\title{
Odor Exposure Causes Central Adaptation and Morphological Changes in Selected Olfactory Glomeruli in Drosophila
}

\author{
Jean-Marc Devaud, Angel Acebes, and Alberto Ferrús \\ Instituto Cajal, Consejo Superior de Investigaciones Cientificas, Madrid E-28002, Spain
}

In an attempt to correlate behavioral and neuronal changes, we examined the structural and functional effects of odor exposure in Drosophila. Young adult flies were exposed to a high concentration of the selected odor, usually benzaldehyde or isoamyl acetate, for $4 \mathrm{~d}$ and subsequently tested for their olfactory response to a variety of odorants and concentrations. The behavioral response showed specific adaptation to the exposed odor. By contrast, olfactory transduction, as measured in electroantennograms, remained normal. In vivo volume measurements were performed on olfactory glomeruli, the anatomical and functional units involved in odor processing. Preexposed flies exhibited volume reduction of certain glomeruli, in an odor-selective manner. Of a sample of eight glomeruli measured, dorsal medial (DM) 2 and ventral (V) were affected by

A striking property of nervous systems is their ability to adapt structural and functional features to the input they receive during lifetime. Behavioral changes, including learning and memory, correlate with modulation of neuronal activity that can eventually lead to changes in gene expression and synapse number (Bailey and Kandel, 1993; Martin and Kandel, 1996; Milner et al., 1998; Corriveau, 1999; Yuste and Sur, 1999). Experience-related changes are usually detected in response to complex environments, however, making it difficult to establish a direct correlation between synapse modification and storage of specific information (for review, see Moser, 1999). The brain of the insect imago undergoes experience-dependent modifications (Bulloch and Ridgway, 1989). Integration centers such as the mushroom bodies (Heisenberg, 1998) and the central complex (Davis, 1996; Strausfeld, 1999) have been well studied in this respect (Brandon and Coss, 1982; Withers et al., 1993; Durst et al., 1994; Gronenberg et al., 1996; Barth and Heisenberg, 1997; Fahrbach et al., 1998; Barth, 1999). Additional brain structures also undergo behaviordependent changes, in particular the antennal lobes (AL), the insect olfactory centers (Masson and Mustaparta, 1990). In the honeybee, shifting to new behavioral tasks in the hive is accompanied by specific AL structural changes, albeit the triggering

\footnotetext{
Received Jan. 29, 2001; revised May 30, 2001; accepted May 30, 2001.

This work was supported by Grants PM99-099 from the Ministry of Technology and 8.5/43/1998 from the Comunidad Autónoma de Madrid (Spain). Postdoctoral fellowships were from the Fondation Fyssen and the Spanish Ministry of Culture and Education (J.M.D.) and by the Comunidad Autónoma de Madrid (A.A.). We appreciate the technical assistance in confocal microscopy of Concha Bailón and Carmen Hernández. We also thank María-Carmen Álvarez for her help in behavioral experiments. Laboratory members and Institute colleagues provided critical comments.

Correspondence should be addressed to A. Ferrús, Instituto Cajal, Consejo Superior de Investigaciones Científicas, Avenida Doctor Arce 37, 28002 Madrid, Spain. E-mail: aferrus@cajal.csic.es.

Copyright (C) 2001 Society for Neuroscience $\quad 0270-6474 / 01 / 216274-09 \$ 15.00 / 0$
}

benzaldehyde exposure, whereas DM6 was affected by isoamyl acetate. Estimation of the number of synapses indicates that volume reduction involves synapse loss that can reach $30 \%$ in the $\mathrm{V}$ glomerulus of flies adapted to benzaldehyde. Additional features of odorant-induced adaptation, including concentration dependence and perdurance, also show correlation, because both effects are elicited by high odor concentrations and are long-lasting (>1 week). Finally, the dunce mutant fails to develop behavioral adaptation as well as morphological changes in the olfactory glomeruli after exposure. These neural changes thus appear to require the cAMP signaling pathway.

Key words: adaptation; olfactory glomeruli; memory; synapse number; dunce; Drosophila stimulus remains unknown (Winnington et al., 1996; Sigg et al., 1997).

The relevance of sensory experience in the young adult for remodeling brain centers and shaping behavior raises the issue of the underlying cellular processes. Drosophila mutants with defects in neural and behavioral plasticity have revealed some of the intracellular signaling pathways involved (for review, see Dubnau and Tully, 1998; Cardin and Abel, 1999). For example, the cAMP cascade is critical for several forms of learning (Kyriacou and Hall, 1984; Tully and Quinn, 1985; Engel and Wu, 1996) and for fine neuronal morphology (Corfas and Dudai, 1989; Davis et al., 1996; Barth and Heisenberg, 1997; Barth, 1999). Most data establishing CAMP regulatory action on the activity dependence of transmission and the number of synaptic varicosities were obtained from the larval neuromuscular junction (Zhong and $\mathrm{Wu}$, 1991, 1993; Schuster et al., 1996; Cheung et al., 1999). Consequently, their relevance to the central events that elicit behavior is difficult to evaluate. Experimental systems are needed in which both behavioral and structural changes can be correlated to test the implication of signaling pathways in these aspects of neural function.

Here we have modified the olfactory environment of adult Drosophila and analyzed the behavioral and anatomical consequences. We find that the behavioral response to subsequent presentation of the same odor is reduced without loss of peripheral sensitivity, a phenomenon to which we refer throughout this report as central adaptation. In addition, specific glomeruli in the AL exhibit a marked size reduction that includes synaptic loss as an underlying mechanism. Behavioral and anatomical effects correlate in several important features, including their onset time and odorant specificity. Furthermore, both effects are absent in dunce mutants, which indicates that the cAMP pathway is impli- 
cated in the structural and functional consequences of odor exposure.

\section{MATERIALS AND METHODS}

Fly stocks and culture. The ENG3 strain was obtained from Dr. M. Ramaswami (University of Arizona, Tucson, AZ). This transgenic line expresses the green fluorescent protein (GFP) associated with the presynaptic protein $n$-synaptobrevin, under the control of the panneural promoter elav (Estes et al., 2000). Experiments were performed on $w$; elav-nsyb-GFP/elav-nsyb-GFP homozygous females. All flies were reared on a standard yeast medium, at $25^{\circ} \mathrm{C}$. The mutant stock $d n c^{1} / d n c^{1}$ was provided by the Drosophila stock center in Umeå (Sweden).

Odor exposure. Adult flies aged $1 \mathrm{~d}$ were transferred to glass bottles containing fresh medium, and a perforated $2 \mathrm{ml}$ eppendorf vial containing $1 \mathrm{ml}$ of the solution used for exposure was attached to the cap. At the end of the fourth day of exposure, unanesthesized flies were collected individually with a mouth aspirator, and males and females were separated. Groups of $\sim 25$ females were transferred into individual vials containing moist filter paper and kept in the dark overnight. All treatments and experiments were performed at room temperature. Odorants used for exposure were dissolved in paraffin oil at dosages indicated as dilution factors. All odors were of the highest purity available from Fluka (Steinheim, Germany), except isoamyl acetate that was from Sigma (St. Louis, MO). For a given exposure protocol and genotype, two groups of flies were prepared, those exposed to the odorant (pre-exposed flies) and those exposed to paraffin oil alone (control flies).

Behavioral assay. The behavioral response of control and pre-exposed flies to different odors was tested using an olfactory $\mathrm{T}$ maze, adapted from Tully and Quinn (1985). In this apparatus, groups of $\sim 25$ females were able to choose between two compartments, each containing a piece of filter paper $(4 \times 1 \mathrm{~cm})$ impregnated with $100 \mu$ l of either the test odor solution (odor compartment) or pure paraffin oil (control compartment). As odor- and oil-containing compartments we used disposable vials whose relative position in the $\mathrm{T}$ maze was at random. The use of impregnated paper, instead of air flows as in the "classical" protocol, was justified by the necessity to perform a great number of tests, and thus to avoid the fine and time-consuming manipulation of air flows. The duration of the test was $30 \mathrm{sec}$. In preliminary experiments, this time was shown to be sufficient for flies to make a choice and retain it, in accordance with Störtkuhl et al. (1999). The response index (RI) (Ayyub et al., 1990) was calculated as the number of flies trapped in the odor compartment minus that of flies trapped in the oil compartment, and divided by the total. Flies trapped in the central compartment were counted, but not included in the calculation. This index values range between 1 (all animals in the odor compartment: complete attraction) and -1 (all animals in the oil compartment: complete repulsion). A value of 0 indicates indifference to the odor tested. For statistical comparisons, RI values were averaged over 30 tests for each experimental condition. Tests in which the nonresponding flies (those trapped in the middle compartment) were more than one-third of the total, were discarded. Each group of flies was tested only once to prevent possible effects on peripheral adaptation. All tests were run in the dark, with the odor and solvent-containing compartments in a horizontal position. Unless otherwise specified, tests were performed on the day after exposure. Groups of flies from each experimental class, however, were tested on different days along several weeks to minimize weather environmental effects.

Electroantennograms. Recordings were performed as previously described (Ayer and Carlson, 1992). Flies were immobilized in a $200 \mu \mathrm{l}$ pipette tip without previous anesthesia. The recording electrode, an unbroken pulled glass electrode ( $\sim 1 \mu \mathrm{m}$ diameter $)$, was leaned on the cuticle of the third antennal segment, and the reference electrode was inserted into the hemolymph, near the proboscis. The electrode was filled with Drosophila Ringer's solution (in mM: $100 \mathrm{NaCl}, 5 \mathrm{KCl}, 20$ $\mathrm{MgCl}_{2}, 0.15 \mathrm{CaCl}_{2}, 5 \mathrm{HEPES}, 115$ sucrose, and 5 trehalose). Odorant stimulation was delivered by an electronic pump device in a continuous nitrogen flow of $0.2 \mathrm{l} / \mathrm{min}$ through a 1 -mm-diameter tube terminating 1 $\mathrm{cm}$ from both antennae. Nitrogen, which does not induce any electrophysiological response by itself (Dubin et al., 1995) was preferred to air to avoid possible stimulation of antennal receptors by carbon dioxide, because in other Diptera, peripheral responses induced by $\mathrm{CO}_{2}$ have been reported (Todd and Baker, 1999). An electrically controlled valve allowed the replacement of this nitrogen flow by odorant pulses while keeping mechanical stimulation in the antenna constant. For each individual test, responses were recorded to five $300 \mathrm{msec}$ pulses at $30 \mathrm{sec}$ intervals of paraffin oil alone or the odorant solution, which were ana- lyzed on pClamp 6.0 software (Axon Instruments, Foster City, CA). No significant variations of response amplitude were observed for any given antenna over successive pulses, indicating that the continuous nitrogen flow had no effect on olfactory transduction. Recordings were obtained from animals from groups that showed behavioral adaptation (RI values of $\sim 0.0$ ). In general, failure to adapt (RI close to control values) was found in approximately one-fourth of the 30 experimental groups. Recordings were performed blind with regard to the experimental treatment (i.e., exposure to oil or to odor).

Confocal microscopy and estimation of glomerular volume. After assessing the behavioral response of ENG3 flies, their brains were dissected in phosphate buffer and mounted unfixed on slides onto which two $20 \times 20$ $\mathrm{mm}$ coverslips had previously been glued to avoid squashing the brains. The preparations were observed as whole mounts on a Leica (Heidelberg, Germany) TLS4D confocal microscope equipped with an inverted microscope (Leitz DMIRB). The constitutive GFP signal was detected by a fluorescein filterblock, with the krypton-argon laser light source set for excitation at $488 \mathrm{~nm}$. Serial optical sections $(512 \times 512$ pixels $)$ were taken at $1 \mu \mathrm{m}$ intervals using a $40 \times$ oil immersion objective and saved as three-dimensional stacks covering the entire AL volume. A quantification software (Q500 MC; Leica) was used for subsequent treatment of files. Stacks were generated by overlaying every fifth image within each AL. The contours of the whole AL neuropil and the selected glomeruli were drawn on these 5- $\mu \mathrm{m}$-thick composite images. Glomeruli were identified on the basis of the three-dimensional atlas of Laissue et al. (1999). For each image series, the areas of the individual contours were summed to calculate the total areas of the structures delimited, which served to estimate the corresponding volumes by multiplying them by image thickness $(5 \mu \mathrm{m})$. All drawings and calculations were run blind respective of the experimental treatment.

Transmission electron microscopy. After the behavioral test on ENG3 flies, brains were dissected and fixed (2.5\% glutaraldehyde and $1 \%$ paraformaldehyde in $0.1 \mathrm{M}$ phosphate buffer, $\mathrm{pH} 7.2$ ) overnight at $4^{\circ} \mathrm{C}$. After two washes in $0.1 \mathrm{M}$ phosphate buffer, they were post-fixed $(1 \%$ OsO4 in $0.1 \mathrm{M}$ phosphate buffer) for $90 \mathrm{~min}$ at $4^{\circ} \mathrm{C}$ in the dark. Brains were then washed twice in buffer, dehydrated in a graded ethanol series, and included in Araldite resin. Silver sections $(60-70 \mathrm{~nm})$ were obtained from the ventral (V) glomerulus in a frontal orientation, using a Reichert Ultracut E ultramicrotome. They were collected on Formvar-coated, single-slot grids, and subsequently stained with uranyl acetate and lead citrate. Observations were performed in a JEOL 1200 EX electron microscope. Synapse quantification was performed blind, on electron micrographs taken within the $\mathrm{V}$ glomerulus, using the disector stereological technique (Sterio, 1984; West, 1999). Alternate ultrathin sections were established as the suitable distance between disectors, because a synaptic specialization never spans beyond two adjacent sections (140 $\mathrm{nm})$. On average, 20 disectors were used in each $\mathrm{V}$ glomerulus, which represent $2.8 \mu \mathrm{m}$ in the anterior-posterior axis or $\sim 20 \%$ of total glomerular volume. Only clearly identifiable T-shape densities surrounded by vesicles were considered as a criterion for synapse.

Statistical analyses. All results are given in the text as mean \pm SEM. Student's $t$ test was used for pairwise comparisons of behavioral data, whereas a Mann-Whitney $U$ test was applied to measurements of glomerular volume and density of synapses. All statistical analyses were done with the SPSS 8.0 software.

\section{RESULTS}

\section{Exposure induces odor-specific adaptation}

To determine whether the exposure affected the behavioral response to odors, we tested control and exposed flies the day after the end of the exposure period (see Materials and Methods). Six-day-old control flies that had been exposed to paraffin oil during days 2-5 show a normal response to $10^{-3}(\mathrm{v} / \mathrm{v})$ benzaldehyde (BZD), i.e., moderate repulsion, as indicated by an RI value of $-0.28 \pm 0.05$ (Fig. 1). This value is identical to that observed for Canton-S females in previous non-exposure experiments $(-0.26 \pm 0.09)$. This coincidence indicates that ENG3 flies (w; elav-nsyb-GFP(elav-nsyb-GFP) do not differ from the standard CS reference strain in this behavior. As control in each experiment, we used ENG3 flies siblings to the experimental group. Both groups of flies are thus cultured in identical conditions of temperature, light, and population density and are of the same age. 

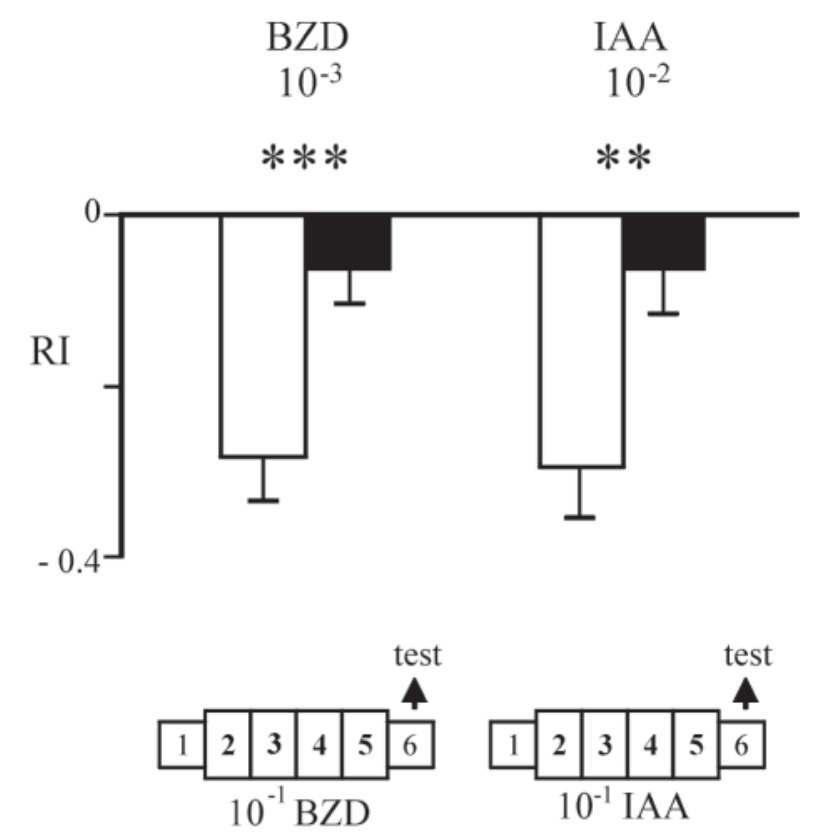

Figure 1. Adaptation of olfactory response after odor exposure. ENG3 flies were exposed to $10^{-1}(\mathrm{v} / \mathrm{v})$ benzaldehyde $(B Z D)$ or isoamyl acetate (IAA) (black bars) or to paraffin oil (white bars) for days 2-5 after eclosion and tested with $10^{-3} \mathrm{BZD}$ and $10^{-2}$ IAA, respectively, the next day in the T-maze apparatus. Note that the change of response leads to indifference to the test odor, corresponding to RI values close to 0. Each average RI value is based on 30 tests of 25 flies each. ${ }^{* *} p<0.01$; ${ }^{* * *} p<$ 0.005 (Student's $t$ test).

Furthermore, to avoid possible effects of ambient conditions and physiological state of the animals, control and experimental groups were tested in parallel. The exposure of controls to paraffin oil was designed to discard possible effects of the manipulation needed to introduce the odor source in the bottles. Opposite to controls, ENG3 flies of the same age (6 d) that had been pre-exposed to $10^{-1} \mathrm{BZD}$ on days 2-5 after eclosion were much less repelled by the odorant. Their mean RI $(-0.06 \pm 0.04)$ indicated that they had become almost indifferent to the odor (Fig. 1). This effect on the behavioral response is statistically significant ( $t=3.25 ; p=0.002$; Student's $t$ test).

To determine whether this effect could be obtained by exposure to odors other than BZD, we repeated the experiment using isoamyl acetate (IAA). Again, exposure of ENG3 flies to $10^{-1}$ IAA significantly altered their response to that odor (control $\mathrm{RI}=-0.29 \pm 0.06$; pre-exposed $\mathrm{RI}=-0.06 \pm 0.05 ; t=2.89 ; p=$ 0.005 ) (Fig. 1). To assess possible motor impairment of preexposed flies, we calculated the proportion of flies that did not respond (i.e., located in neither of the two compartments at the end of the test) for each experimental condition. If the change in response were attributable to some unknown effect of exposure on locomotion, a significant increase in this proportion would be expected. In none of the cases, control and pre-exposed flies yielded a significant difference (data not shown), suggesting that exposure does not influence locomotor activity. We refer to this behavioral effect as "adaptation." The effect, thus, is defined as a group response and not as an individual feature. On average, approximately one-fourth of the tested groups in any given data point failed to show evidence of adaptation.

To test the specificity of this phenomenon, flies exposed to either BZD or IAA were tested to three additional odors: one of the same functional group [either butaldehyde (BA) or ethyl
A B
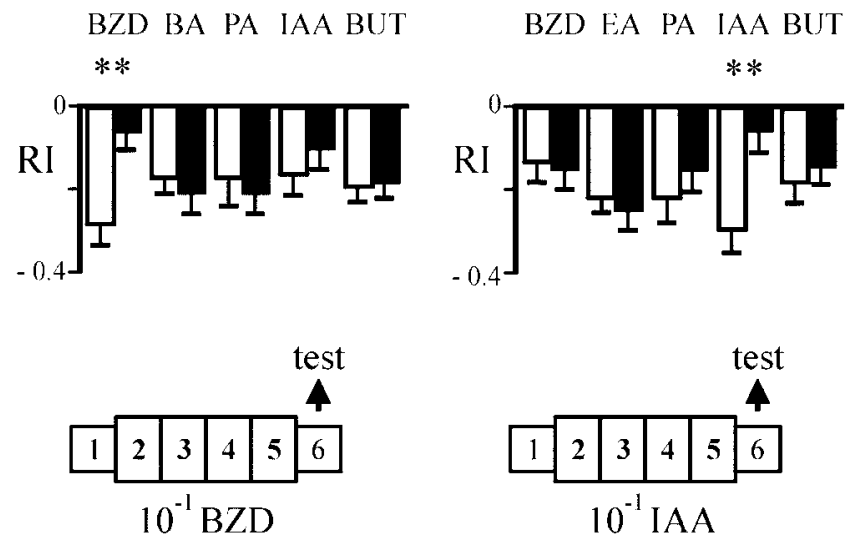

Figure 2. Adaptation is odor-specific. A, ENG3 flies were exposed to $10^{-1}$ benzaldehyde (black bars) or to paraffin oil (white bars) and tested with one of the following odors: $10^{-3}$ benzaldehyde $(B Z D), 10^{-2}$ butaldehyde $(B A), 10^{-2}$ propionic acid $(P A), 10^{-2}$ isoamyl acetate $(I A A)$, and $10^{-3}$ butanol $(B U T)$. $B$, Equivalent test after exposure to $10^{-1}$ isoamyl acetate $(I A A)$ using $10^{-2}$ ethyl acetate $(E A)$ instead of BA as test odorants. $n=30$ tests in each case. ${ }^{* *} p<0.01$ (Student's $t$ test).

acetate (EA)] and two with different functional groups [propionic acid (PA) and butanol (BUT)] (Fig. 2). These odors were tested at concentrations previously shown to elicit avoidance levels similar to those of BZD and IAA, as shown by control responses. The results show that flies previously exposed to either BZD (Fig. $2 A$ ) or IAA (Fig. $2 B$ ) display a response significantly different from that of their respective controls only when tested with the odor to which they have been exposed. With this sample of odorants, thus, no cross-adaptation was detected.

\section{Exposure-induced adaptation is a dose-dependent and long-lasting phenomenon}

To characterize this form of adaptation, we modified several parameters in the standard protocol (Fig. 3). Dose-of-exposure dependence was first tested. Flies were pre-exposed to various dilutions of BZD or IAA and tested as previously $\left(10^{-3}\right.$ for BZD or $10^{-2}$ for IAA). As shown in Figure $3 A$ for BZD, the mean RI of pre-exposed flies was significantly different from control flies only when high concentrations $\left(10^{-1}\right.$ and $\left.10^{-2}\right)$ had been used for exposure $(t=2.50, p=0.015$ and $t=2.54, p=0.014$, respectively). The behavioral effect of odor exposure is thus a dose-dependent effect that requires high doses of the stimulus.

Second, we assayed the dose-of-test without varying the doseof-exposure $\left(10^{-1}\right)$. The experiment was performed with BZD and IAA, yielding quite similar results. As shown for BZD (Fig. $3 B$ ), control flies display a typical dose-response profile as that of wild-type flies; that is, indifference at low doses $\left(10^{-5}\right)$, low attraction at moderate concentrations $\left(10^{-4}\right)$, and avoidance at high concentrations $\left(10^{-3}\right.$ to $10^{-1}$ in these experiments) (Alcorta, 1991; Acebes and Ferrús, 2001). In contrast, pre-exposed flies show a flattened curve, with lower levels of attraction and repulsion. Statistically significant differences were found for the $10^{-3}, 10^{-2}$ and $10^{-1}$ dilutions $(t=2.16, p=0.035 ; t=4.32, p<$ 0.001 ; and $t=2.16, p=0.035$, respectively). A similar curve was obtained for IAA (data not shown), with a significant shift of RI values at $10^{-2}$ and $10^{-1}(p=0.005$ in both cases). Olfactory 
A

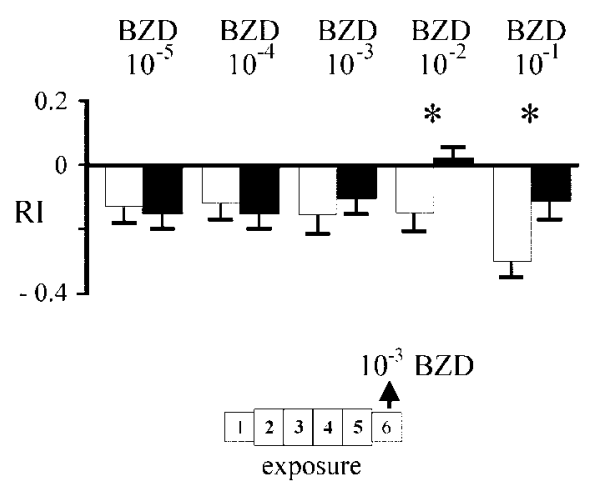

B
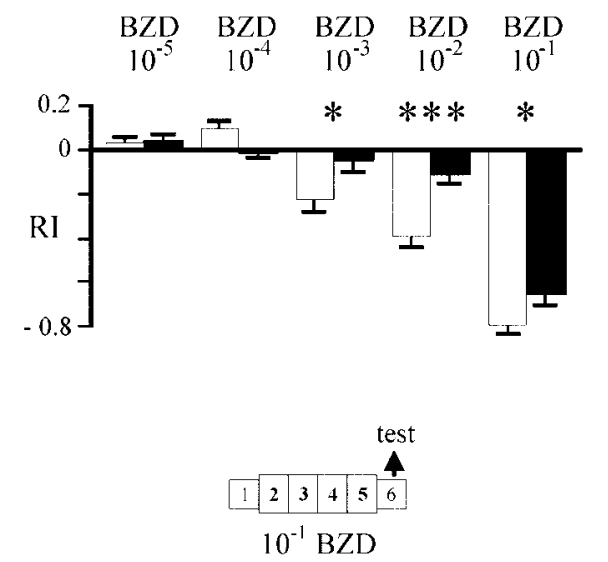

C
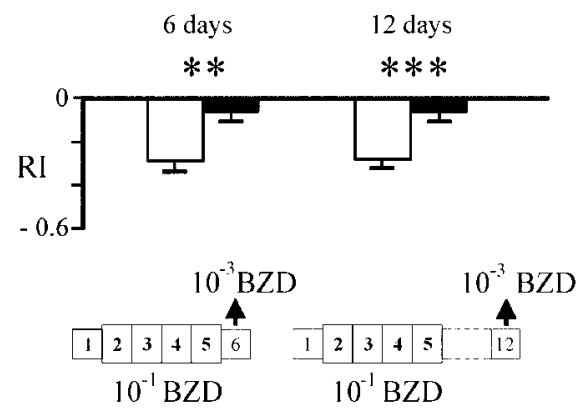

Figure 3. Behavioral parameters of odor exposure. A, Dose effect of odor exposure. ENG3 flies were exposed to various concentrations of BZD (black bars) or paraffin oil (white bars) and tested with $10^{-3}$ BZD. Note that significant adaptation is detected at high concentrations only. $B$, Dose effect of test odor. ENG3 flies were exposed to $10^{-1}$ BZD or paraffin oil and tested with variable BZD concentrations (between $10^{-1}$ and $10^{-5}$ ). $C$, Perdurance of exposure induced adaptation. ENG3 flies were exposed to $10^{-1} \mathrm{BZD}$ or paraffin oil and tested at several ages: on day $6(1 \mathrm{~d}$ after the end of the exposure period) and on day 12 (7 d after exposure). ${ }^{*} p<$ $005 ;{ }^{* *} p<0.01 ; * * p<0.005$ (Student's $t$ test).

adaptation thus manifests as a decreased response (RI values closer to 0 ), and it is most prominent for concentrated stimuli.

Third, to determine whether the behavioral change observed is a short- or long-term effect, we tested pre-exposed flies on day 12 , 1 week after the end of exposure (Fig. $3 C$ ). At this time, adaptation was still evident with respect to controls $(t=3.38 ; p=0.001)$.
Table 1. Amplitude of extracellular potentials recorded on the antennae (EAGs)

\begin{tabular}{lll} 
& \multicolumn{2}{l}{ EAG amplitude $(\mathrm{mV})$} \\
\cline { 2 - 3 } Stimulant odor & Control & Preexposed \\
\hline Paraffin oil & $2.62 \pm 0.13$ & $2.61 \pm 0.27$ \\
$10^{-3}$ BZD & $6.19 \pm 0.36$ & $5.96 \pm 0.31$
\end{tabular}

$\overline{\text { Electroantennograms were recorded on ENG3 flies on the day after the end of }}$ exposure. Flies had been exposed to either paraffin oil (control) or BZD $10^{-1}(\mathrm{v} / \mathrm{v})$ (preexposed) for 4 d. $n=9$ in all cases.

The adapted response $(-0.06 \pm 0.04)$ was identical to that of flies tested on the day after exposure $(-0.06 \pm 0.05)$. The perdurance of the behavioral effect of exposure therefore, appears to be $>1$ week.

\section{Adapted flies have a normal sensory response}

The diminished reactivity of pre-exposed flies could be attributable to sensory adaptation as in short-term exposure experiments (Störtkuhl et al., 1999). This possibility was tested by performing electroantennograms (EAGs) at the time when behavioral tests were usually performed (the day after the end of exposure). Flies exposed to $10^{-1} \mathrm{BZD}$ that had displayed adaptation to $10^{-3}$ BZD showed no significant differences in their EAG amplitudes with respect to controls $(p>0.05)$ (Table 1). Thus, no peripheral adaptation could be detected under conditions in which the behavioral response was modified by exposure. This observation justifies that the phenomenon described here is considered as central adaptation.

\section{Exposure reduces the volume of selected glomeruli}

Because no exposure-induced peripheral changes were apparent, we looked for possible central effects of the behavioral change in the first olfactory centers, the antennal lobes. The ENG3 line is suitable to visualize glomeruli caused by GFP expression in all synaptic areas (Fig. $4 A$ ). Whole brains of control and pre-exposed flies were observed in confocal microscopy, and serial image stacks from the antennal lobes were compared blind. In a first qualitative evaluation, no consistent difference was detected in AL size or structure, between the two experimental groups. Looking for possible, subtler changes, we performed a quantitative study to estimate the volume of the whole AL neuropil and of several glomeruli. A subset of eight glomeruli was selected for measurement (Fig. 4A), including four of the five most easily identifiable or "principal" glomeruli (Laissue et al., 1999) dorsal (D), dorsal medial (DM) 3, V, ventral anterior (VA) 2. The fifth, VA1, was excluded because it is subdivided into compartments (Laissue et al., 1999) that were not always easily distinguishable from adjacent glomeruli using our visualization method. The other glomeruli selected were DM2, DM6, VA3, and VA4.

Comparison between control and pre-exposed flies $\left(10^{-1}\right.$ BZD) showed no significant differences in the volume of the whole antennal lobe neuropil (data not shown). When comparing values from individual glomeruli, however, significant differences were found. Two glomeruli, DM2 and V, showed a strong volume reduction in pre-exposed flies with respect to controls (Fig. 4B). The relative decrease was of $14 \%$ for DM2 and $33 \%$ for $\mathrm{V}(U=$ 23.0, $p=0.028$ and $U=21.0, p=0.002$, respectively; MannWhitney $U$ test). None of the other glomeruli measured show significant change ( $p>0.1$ for all pairwise comparisons). 

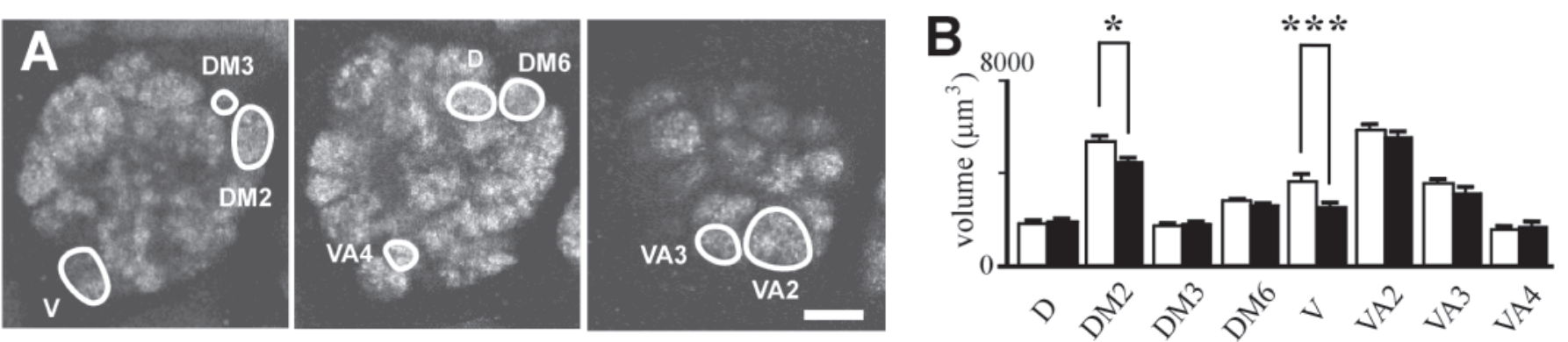

Figure 4. Structural effects of adaptation: glomerular volumes. A, Frontal view of three representative stacks, each composed of five confocal images, taken from the right AL of a 6-d-old ENG3 fly. Glomeruli are visible by the expression of the neuronal synaptobrevin-GFP construct. The glomeruli selected for measurement are outlined and labeled. Scale bar, $25 \mu \mathrm{m}$. B. Estimated volume of the selected glomeruli. ENG3 flies were exposed to $10^{-1}$ benzaldehyde (black bars) or to paraffin oil (white bars) during days 2-5, and their brains were examined on the next day. $V$, Ventral, $V A$, ventral anterior; $D$, dorsal; $D M$, dorsal medial. * $p<0.05$; ${ }^{* *} p<0.005$ (Mann-Whitney $U$ test). An average of 10 glomeruli were measured in each group.
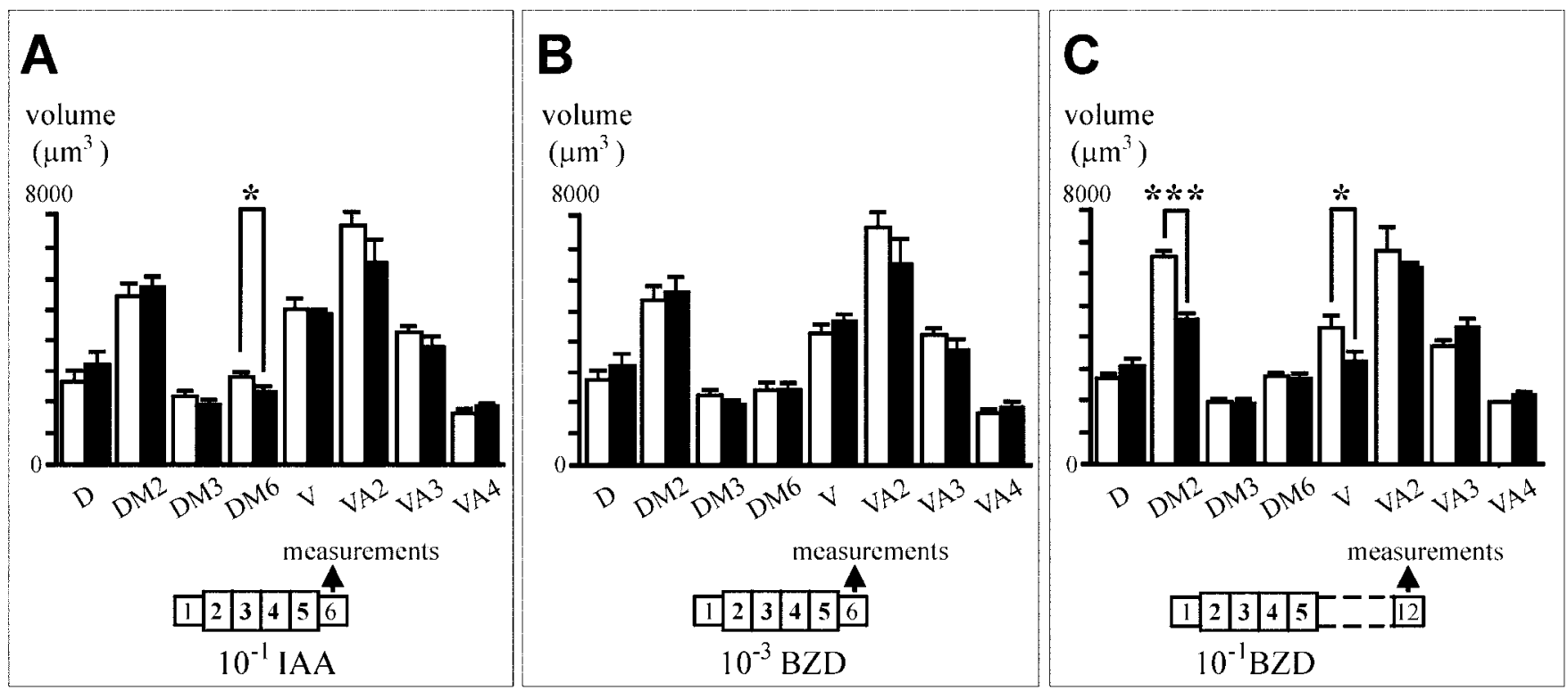

Figure 5. Structural parameters of odor exposure. A, Specificity of affected glomeruli. ENG3 flies were exposed to IAA $10^{-1}$ (black bars) or to paraffin oil (white bars), and their brains were examined on the next day. Note that DM6 is affected, whereas DM2 and V remain normal. B, Dose effect on glomerular volume. The same procedure was applied using BZD $10^{-3}$ as the odor of exposure. Note the absence of significant changes at this concentration of exposure odorant, which is also ineffective to produce adaptation (Fig. $3 A$ ). $C$, Time extent of the structural changes. ENG3 flies were exposed to $10^{-1} \mathrm{BZD}$ or paraffin oil, and their brains were examined 1 week after the end of exposure, i.e., on day 12 of adulthood. * $p<0.05$; *** $p<$ 0.005 (Mann-Whitney $U$ test). An average of 10 glomeruli were measured in each group.

\section{Reduction in glomerular volume is odor-specific, dose- dependent, and long-lasting}

The specificity of this morphological change was evaluated by a further volumetric study on flies pre-exposed to IAA $\left(10^{-1}\right)$ rather than to BZD (Fig. 5A). In this case, only one glomerulus (DM6) was significantly affected, also in the direction of volume decrease, $15 \%$, in pre-exposed flies $(U=33.0, p=0.040$; MannWhitney $U$ test). Interestingly, IAA treatment had no effect on the volumes of DM2 and $\mathrm{V}(p>0.1$, as for other glomeruli), nor was the total neuropil volume affected. Exposure to odors thus induced a significant reduction in volume of individual glomeruli, but those affected differed according to the odor used.

The possible dose dependence of the glomerular volume changes was also studied. After exposure to a BZD concentration insufficient to induce adaptation $\left(10^{-3}\right)$ (Fig. $3 A$ ), we estimated the volumes of the same set of glomeruli (Fig. $5 B$ ). None of the eight glomeruli showed significant volume changes between control and pre-exposed flies, nor did the volume of the neuropil ( $p>0.1$ for all pairwise comparisons; Mann-Whitney $U$ test). The anatomical effect of exposure, as shown previously for adaptation, therefore appears to depend on the stimulus concentration.

Finally, we analyzed whether long-term maintenance of adaptation was accompanied by a similarly long-lasting structural modification. Measurements were performed on control and preexposed flies $\left(10^{-1}\right.$ BZD) 1 week after the end of exposure (Fig. $5 C$ ). Under these conditions, glomeruli DM2 and V still exhibited a decreased volume in pre-exposed flies (DM2: $-30 \%, U=0.0$, $p=0.000 ; \mathrm{V}:-25 \%, U=36.0, p=0.025)$, whereas the remaining glomeruli remained unaffected $(p>0.1)$. The extent of this volume reduction thus seems to last $>1$ week, as does the behavioral adaptation.

\section{Synapse number decreases in reduced glomeruli of pre-exposed flies}

Because the volume estimations were obtained from the synaptic expression of GFP in glomeruli, the volume decrease of DM2 and 

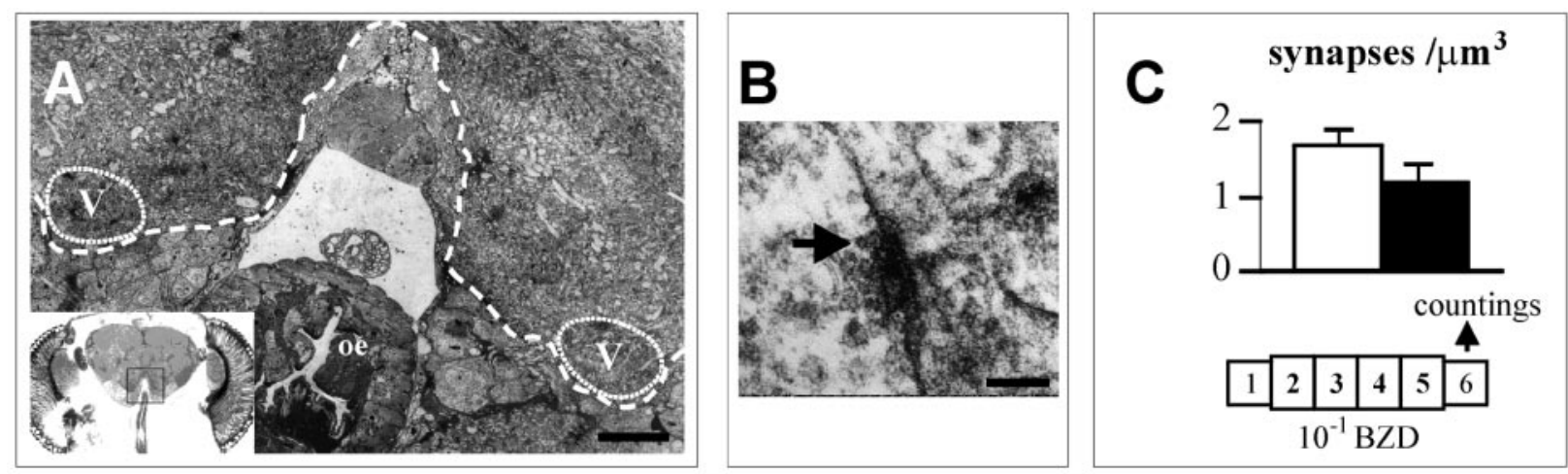

Figure 6. Density of synapses in exposed flies. $A$, Semithin section of $\mathrm{AL}$ in frontal view covering the regions marked in the inset. The limits of the glomerular neuropils are indicated by a dotted line. The $\mathrm{V}$ glomerulus can be identified on each side. oe, Esophagus. $B$, High-power view from inside the $\mathrm{V}$ glomerulus showing the characteristic T-shaped presynaptic density (arrow). C, Estimated density of synapses in V glomeruli of ENG3 flies after exposure to either paraffin oil (white bars) or to $10^{-1}$ BZD (black bars). Countings were from four glomeruli in each group of flies, using an average of 20 disectors (see Materials and Methods). Scale bars: $A, 15 \mu \mathrm{m} ; B, 50 \mathrm{~nm}$.

$\mathrm{V}$ was likely to result from synaptic rearrangements. To further document this interpretation, we estimated intraglomerular density of synapses in control and pre-exposed flies following the standard protocol with BZD. If glomerular volume was reduced without change in number of synapses, density values should increase in pre-exposed flies. Alternatively, if synaptic loss occurred, no increase in density should be observed. The electron microscope material for this evaluation was from the $\mathrm{V}$ glomerulus ( $n=4$ in control and experimental flies), because it is the only one that can be identified unambiguously at the ultrastructural level, which is attributable to its prominent position in the ventral side of the antennal lobe (Fig. 6A). The number of disectors used (average of 20 ) represent $\sim 20 \%$ of the total glomerular volume. The difficulty in identifying a clear separation of adjacent glomeruli by glial cells at the ultrastructural level precluded extension of the study to other glomeruli. The general neuropil organization in the $\mathrm{V}$ glomerulus was indistinguishable between control and pre-exposed flies. In particular, we observed no evidence of cellular necrosis, which rules out fiber degeneration as an explanation for the volume reduction in adapted flies. In addition, 6-d-old brains from exposed and control flies were stained by the Nile Blue sulfate method (Teillet et al., 1998) as an assay for cell death. Very few cases of cell death were observed in both groups of flies (data not shown). It seems thus, that excitotoxicity is not a likely cause of the morphological changes observed.

Counting of synapses, identified by their T-shape densities (Fig. $6 B)$ showed that, on the day after exposure, the density $(\sigma)$ tended to be lower in pre-exposed flies than in control flies (Fig. $6 C)$. Nevertheless, the difference was not significant in our sample ( $U=4.0, p=0.343$; Mann-Whitney $U$ test). Considering the reduction of volume $(\Delta \mathrm{V})$ in this glomerulus (Fig. 4), the resulting effect on the number of synapses $(N)$ can be estimated as $\Delta N=$ $\Delta V \times \sigma$. It thus appears that $N$ in the $\mathrm{V}$ glomerulus of flies adapted to $10^{-1} \mathrm{BZD}$ is $\sim 30 \%$ lower than in controls.

\section{dunce mutants do not adapt or modify glomerular volume}

To elucidate the cellular mechanisms acting during odorant exposure that lead to the observed changes in AL morphology and olfactory response, we analyzed the mutant dunce $\left(d n c^{1}\right)$ under the adaptation protocol. The gene encodes a cAMP-dependent phosphodiesterase, PDE II, and the mutant displays higher than normal cellular cAMP levels (Byers et al., 1981). As a result, the mutant is impaired in various forms of learning and memory (for review, see Dubnau and Tully, 1998). In addition, it shows alterations in synaptic function and structure, at least in the larval neuromuscular junction (Zhong and Wu, 1991; Zhong et al., 1992). When tested for their behavioral response to $10^{-3} \mathrm{BZD}$ after exposure to $10^{-1}, d n c^{1} / d n c^{1}$ flies exhibited no significant change in RI value with respect to oil-exposed controls of the same genotype (Fig. 7A) $(t=0.119 ; p>0.05)$. Both groups showed weak avoidance (controls, $-0.18 \pm 0.05$; pre-exposed, $-0.19 \pm 0.06)$, with values that were not significantly different from those exhibited by control ENG3 flies (Figs. 1-3) $(t=1.46$; $p>0.1)$. The olfactory behavior of dunce mutants thus, does not appear to be affected by exposure. Similar results were obtained also in IAA exposure tests in which RI of odor-exposed flies was $-0.16 \pm 0.05$, and that of oil-exposed flies was $-0.18 \pm 0.06(t=$ $0.24 ; p>0.05)$. However, the glomerular volume of $d n c^{1} / d n c^{1}$; ENG3/ENG3 flies exposed to $10^{-1}$ BZD show no significant change in any of the eight measured glomeruli $(p>0.05$ in all cases) (Fig. $7 B$ ). In particular, DM2 and V glomeruli remained unaffected $(U=36.0, p=1.00$ and $U=59.0, p=0.478$, respectively), whereas they were strongly reduced in adapted ENG3 flies (Figs. 4, 5C). The intracellular cAMP level therefore appears to be one of the molecular requirements for the functional and structural changes elicited by odor exposure. In turn, dunce flies serve as an additional control for the morphological and behavioral changes observed in ENG3 flies.

\section{DISCUSSION}

This study shows that long-term exposure of young Drosophila adults to a concentrated odor produces olfactory adaptation. Exposure causes a parallel decrease in the volume of specific glomeruli, in which synapse loss appears to occur. Taken together, the data correlate structural brain plasticity and behavioral adaptation, thus providing experimental evidence for a possible link between them.

\section{Peripheral versus central adaptation}

The observed change in olfactory response in pre-exposed flies represents an example of behavioral change reminiscent of, but different from, that induced by a much shorter exposure time (1 min) (Störtkuhl et al., 1999). Short-term exposure induces sensory adaptation in the antenna, sufficient to decrease the behav- 


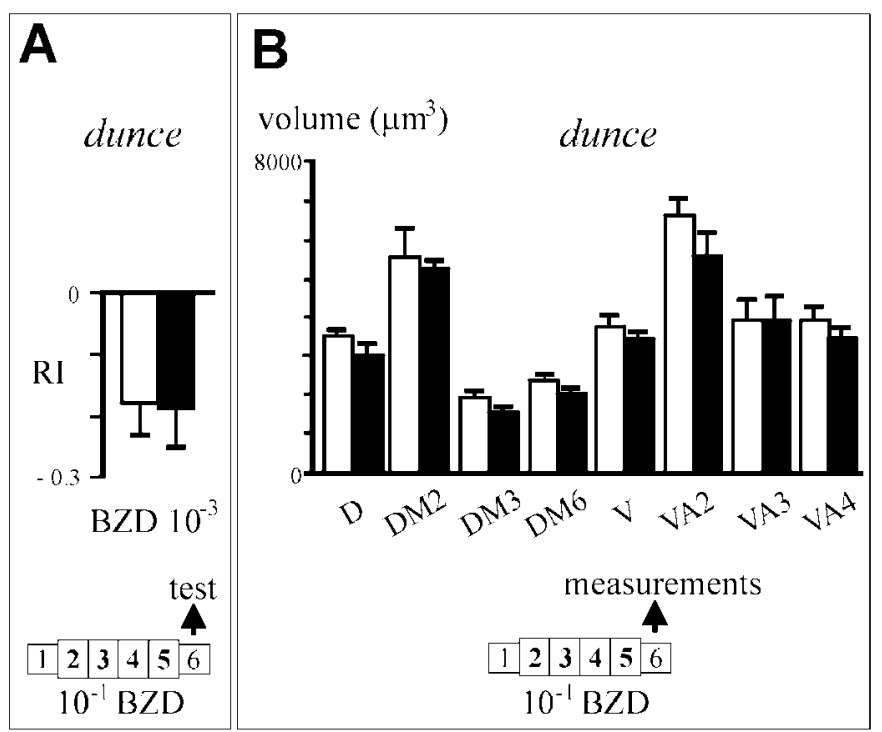

Figure 7. Odor-exposed dunce mutants do not show adaptation or changes of glomerular volume. $A$, Behavioral response to $10^{-3} \mathrm{BZD}$ of $d n c^{1} / d n c^{1}$ flies previously exposed either to paraffin oil (white bars) or BZD $10^{-1}$ (black bars). B, Estimations of glomerular volume in $d n c^{1} /$ $d n c^{1}$; ENG3/ENG3 flies (same exposure protocol). An average of 10 glomeruli were measured in each group.

ioral response. Although sensory adaptation is likely to occur during long exposure periods, its short recovery time and lack of specificity would be incompatible with the observations reported here. The remarkable temporal stability seen under these conditions (at least 1 week) contrasts with the rapid extinction, within minutes, of the peripheral effect of short exposure. Indeed, flies adapted under our protocol had normal EAG amplitudes at the time when the behavioral change was observed. Moreover, the effect of long exposure is odorant-selective, because no crossadaptation was found comparable to that shown previously for short exposure times. This feature is particularly relevant because our sample of odors included most of those used in that report. In addition, odorants of the same functional group as those used for exposure (aldehydes and esters) did not yield cross-adaptation. Sensory adaptation thus cannot account for the behavioral effect described here, which must consequently take place in integrative brain centers.

\section{Central adaptation versus other behavioral changes}

The behavioral effect of odor exposure is unlikely to result from associative conditioning between odor and food. Being continuously present while the animals feed, the odor lacks the predictive value required for association. Even if flies had associated the odor with a positive reinforcement, pre-exposed flies would be expected to be more attracted to the odor. Sandoz et al. (2000) reached similar conclusions after observing reduced avoidance in odor exposed bees, whereas animals positively conditioned to the same odor showed attraction instead. Furthermore, Manning (1967) found that, even when exposed without food, flies still exhibited what she called "habituation." As we have been unable to produce dishabituation so far, we use the term "central adaptation" for the nonassociative effect of long-term odor exposure. Limited data suggest that the behavioral effect persists 2 weeks after exposure. At this time, however, it becomes difficult to discriminate between long-lasting adaptation and age-dependent effects in control animals. Considering the behavioral and struc- tural features of the phenomenon described here for Drosophila, it appears reminiscent of "imprinting" as described in some vertebrates (for review, see Rose and Stewart, 1999). A critical time window in development, however, remains to be established before a comparison between both phenomena can be made.

Although the experimental treatment used here may appear extreme, it is not far from natural conditions. First, it should be noted that the concentrations used for exposure are higher than those actually perceived by the animals because of diffusion in the bottle. Second, although it is clear that animals in the open field are unlikely to be exposed to pure odorants, it is not unreasonable to think that ambient aromas, in which the animals spend most of their time, are likely to produce long-lasting effects. In the case of Drosophila, pioneer studies (Hoffmann 1985) had already shown modulation of olfactory behavior by natural odorants produced by the medium (fruits) on which the animals had grown.

\section{Glomerular specificity of experience-dependent changes}

The behavioral adaptation reported here correlates with changes in glomerular volume. Although behavioral responses and volume estimations vary from one experiment to another, comparisons between pre-exposed and control groups tested in parallel show that exposure acts on both parameters in a similar manner in terms of odor specificity, dose dependence, and time duration.

Structural data have been obtained from eight glomeruli, including analysis of density of synapses for one of them. A general effect in the whole AL appears unlikely. First, pre-exposed animals responded normally to the stimuli assayed, and second, most glomeruli measured exhibited no significant change in volume after exposure. Emphasis must be made on the significance of the glomerulus-specific changes, despite interindividual variability. For example, the mean deviation of the average volume of $\mathrm{V}$ glomeruli in controls was $15 \%$, whereas the reduction in BZDadapted flies was $33 \%$. The specificity is consistent with earlier observations in other insects and vertebrates. Identified glomeruli in the honeybee display unique growth patterns during adult life (Winnington et al., 1996) in relation to behavioral tasks in the hive (Sigg et al., 1997). In rodents, postnatal exposure has odorspecific effects on olfactory bulb organization (Pinching and Døving, 1974; Dalland and Døving, 1981; Harvey and Colley, 1986; Laing et al., 1985; Panhuber, 1986). Still, no conclusion could be drawn about whether the regions affected were those stimulated by the exposure odorant or those that had been comparatively less stimulated by other odors (Brunjes and Frazier, 1986; Panhuber, 1986). The data reported here favor the former interpretation, because the response to the odor of exposure was modified regardless of its chemical nature, and the glomeruli affected varied with the odor used. It is plausible that odorants activating the same pathway as the exposure odor would yield crossadaptation. However, the absence of data linking olfactory receptors and glomeruli in Drosophila prevents testing this hypothesis. We find that odorants sharing a functional group with the exposure odor do not produce cross-adaptation. This structural similarity, however, may not be sufficient for the odors to be perceived as "similar" by the animals. Data from other species indicate that the carbon chain length is also a determinant parameter (Rubin and Katz, 1999; Sachse et al., 1999).

The only data available in Drosophila on the functional specificity of glomeruli are difficult to interpret in relation to the glomerular organization as known today. Measurements of $\left[{ }^{3} \mathrm{H}\right]$ 2-deoxyglucose uptake after odor stimulation (Rodrigues 
and Buchner, 1984; Rodrigues, 1988) identified several glomeruli activated by benzaldehyde. The glomerular map then available, however, lacked the detail of the current three-dimensional atlas (Laissue et al., 1999). Only 22 glomeruli had been described then (Pinto et al., 1988), whereas 43 are known today. In addition, the benefits of the ventral location of the $\mathrm{V}$ glomerulus are missed in the horizontal sections used in those studies. Consequently, the glomerular maps used in previous reports render uncertain the identification of the activated glomeruli. In the light of data reported here, V and DM2 glomeruli would be strongly activated by benzaldehyde, but not by isoamyl acetate, whereas DM6 would be a target for IAA stimulation. Further functional studies are needed to validate this hypothesis.

\section{Synaptic rearrangements in the AL and memory}

The electron microscope study suggests that synaptic loss is an underlying mechanism of adaptation. Because the density of synapses in the $\mathrm{V}$ glomerulus does not significantly change, but its volume, $\Delta \mathrm{V}$, does it by $\sim 30 \%$ in pre-exposed flies, the total synapse number should be reduced in a similar proportion. Such modifications would be consistent with previous reports on honeybee glomeruli, in which AL maturation is modulated by the olfactory environment (Gascuel and Masson, 1987, 1991) or activity-dependent changes (Winnington et al., 1996; Sigg et al., 1997). The latter reports show volume increase (rather than reduction) in only one of the bee's glomeruli, T4-2(1), correlated with the shift from hive to foraging behavior. The role of odorants in this process, however, was not identified. In contrast, our results in Drosophila show that changes in glomerular size correlate with quantitative and qualitative aspects of olfactory stimulation.

In the same context, it is known that some forms of olfactory memory can be induced by locally applied treatments to the AL in Apis (Hammer and Menzel, 1998; Müller, 2000). In addition, ALs have been implicated in memory formation during courtship in Drosophila (McBride et al., 1999). In line with all these observations, we suggest that the selective structural modifications of glomeruli that result from olfactory experience may serve as a neural basis for memory. It is plausible that the actual direction of the morphological changes (e.g., increase or decrease of $N$ ) depends on the amount, rather than the type, of stimulus. While this report illustrates a case of reduced sensitivity of perception and a decrease in $N$, an accompanying study (Acebes and Ferrús, 2001) shows a case in which the increment of $N$ in the AL correlates with an increment of olfactory perception. Taken together, both reports document the biological role of $N$, setting the level of stimulus perception.

The regulation of the cAMP cascade appears as a molecular process that sustains the cellular and behavioral changes reported here, as well as others on the structural plasticity in the larval peripheral (Budnik, 1996) and adult CNSs (Balling et al., 1987) reported previously. The dunce mutant, which has chronically elevated intracellular cAMP levels, is deficient in these features, as shown by abnormal fiber numbers in the mushroom bodies (MB) (Balling et al., 1987) and altered volumetric changes of central complex and MB after light exposure (Barth and Heisenberg, 1997; Barth, 1999). The gene is expressed in AL, although at lower levels than in MB (Nighorn et al., 1991). Exposure effects may thus be expected in this brain center as well. Here, glomerular volume remained unaffected by odor exposure, and central adaptation was absent in homozygous $d n c^{1}$ mutants, which are thus impaired for both nonassociative and associative forms of olfactory memory (Dudai et al., 1976; Tempel et al., 1983). The
cAMP cascade is implicated in memory formation in bees AL (Müller, 2000) and in structural and functional modulation of Drosophila larval neuromuscular junction (Zhong and Wu, 1991, 1993; Davis et al., 1996; Cheung et al., 1999). We propose that the cAMP pathway may also lead to structural synaptic remodeling in AL, which would thus be an appropriate feature for long-term memory of the odorant environment.

\section{REFERENCES}

Acebes A, Ferrús A (2001) Increasing the number of synapses modifies olfactory perception in Drosophila. J Neurosci 21:6264-6273.

Alcorta E (1991) Characterization of the electroantennogram in Drosophila melanogaster and its use for identifying olfactory capture and transduction mutants. J Neurophysiol 65:702-714.

Ayer RK, Carlson J (1992) Olfactory physiology in the Drosophila antenna and maxillary palp: acj6 distinguishes two classes of odorant pathways. J Neurobiol 23:965-982.

Ayyub C, Paranjape J, Rodrigues V, Siddiqi O (1990) Genetics of olfactory behavior in Drosophila melanogaster. J Neurogenet 6:243-262.

Bailey CH, Kandel ER (1993) Structural changes accompanying memory storage. Annu Rev Neurosci 55:397-426.

Balling A, Technau GM, Heisenberg M (1987) Are the structural changes in adult Drosophila mushroom bodies memory traces? Studies on biochemical learning mutants. J Neurogenet 4:65-73.

Barth M (1999) Experience-dependent development of the adult optic lobe and central brain in Drosophila melanogaster. Ross Fiziol Zh Im I Sechenova 85:184-194.

Barth M, Heisenberg M (1997) Vision affects mushroom bodies and central complex in Drosophila melanogaster. Learn Mem 4:219-229.

Brandon JG, Coss RG (1982) Rapid dendritic spine stem shortening during one-trial learning: the honeybee's first orientation flight. Brain Res 252:51-61.

Brunjes PC, Frazier LL (1986) Maturation and plasticity in the olfactory system of vertebrates. Brain Res Rev 11:1-45.

Budnik V (1996) Synapse maturation and structural plasticity at Drosophila neuromuscular junction. Curr Opin Neurobiol 6:858-867.

Bulloch AGM, Ridgway RL (1989) Neuronal plasticity in the adult invertebrate system. J Neurobiol 20:295-311.

Byers D, David RL, Kiger JA (1981) Defect in cyclic AMP phosphodiesterase due to the dunce mutation of learning in Drosophila melanogaster. Nature 289:79-81.

Cardin JA, Abel T (1999) Memory suppressor genes: enhancing the relationship between synaptic plasticity and memory storage. J Neurosci Res 58:10-23.

Cheung US, Shayan AS, Boulianne GL, Atwood HL (1999) Drosophila larval neuromuscular junction's responses to reduction of cAMP in the nervous system. J Neurobiol 40:1-13.

Corfas G, Dudai Y (1989) Habituation and dishabituation of a cleaning reflex in normal and mutant Drosophila. J Neurosci 9:56-62.

Corriveau RA (1999) Electrical activity and gene expression in the development of vertebrate neural circuits. J Neurobiol 41:148-157.

Dalland T, Døving KB (1981) Reaction to olfactory stimuli in odorexposed rats. Behav Neural Biol 32:79-88.

Davis RL (1996) Physiology and biochemistry of Drosophila learning mutants. Physiol Rev 76:299-317.

Davis GW, Schuster CM, Goodman CS (1996) Genetic dissection of structural and functional components of synaptic plasticity. III. CREB is necessary for presynaptic functional plasticity. Neuron 17:669-679.

Dubin AE, Heald NL, Cleveland B, Carlson JR, Harris GL (1995) Scutoid mutation of Drosophila melanogaster specifically decreases olfactory responses to short-chain acetate esters and ketones. J Neurobiol 28:214-233.

Dubnau J, Tully T (1998) Gene discovery in Drosophila: new insights for learning and memory. Annu Rev Neurosci 21:407-444.

Dudai Y, Jan Y-N, Byers D, Quinn W, Benzer S (1976) dunce, a mutant of Drosophila melanogaster deficient in learning. Proc Natl Acad Sci USA 73:1684-1688.

Durst C, Eichmüller S, Menzel R (1994) Development and experience lead to increased volume of subcompartments of the honeybee mushroom body. Behav Neural Biol 62:259-263.

Engel JE, Wu C-F (1996) Altered habituation of an identified escape circuit in Drosophila memory mutants. J Neurosci 16:3486-3499.

Estes PS, Ho GL, Narayanan R, Ramaswami M (2000) Synaptic localization and restricted diff usion of a Drosophila neuronal synaptobrevingreen fluorescent protein chimera in vivo. J Neurogenet 13:233-255.

Fahrbach SE, Moore D, Capaldi EA, Farris SM, Robinson GE (1998) Experience-expectant plasticity in the mushroom bodies of the honeybee. Learn Mem 5:115-123.

Gascuel J, Masson C (1987) Influence of olfactory deprivation on synapse frequency in developing antennal lobe of the honeybee Apis mellifera. Neurosci Res Commun 1:173-180. 
Gascuel J, Masson C (1991) Developmental study of afferented and deafferented bee antennal lobes. J Neurobiol 22:795-810.

Gronenberg W, Heeren S, Holldöbler B (1996) Age-dependent and taskrelated morphological changes in the brain and mushroom bodies of the ant Camponotus floridanus. J Exp Biol 199:229-263.

Hammer M, Menzel R (1998) Multiple sites of associative odor learning as revealed by local brain microinjections of octopamine in honeybees. Learn Mem 5:146-156.

Harvey FE, Colley JJ (1984) Effects of external chemical environment on the developing olfactory bulbs of the mouse (Mus musculus). Brain Res Bull 13:541-547.

Heisenberg M (1998) What do the mushroom bodies do for the insect brain? An introduction. Learn Mem 5:1-10.

Hoffmann AA (1985) Effect of experience on oviposition and attraction in Drosophila: comparing apples and oranges. Am Nat 126:41-51.

Kyriacou CP, Hall JC (1984) Learning and memory mutations impair acoustic priming of mating behavior in Drosophila. Nature 308:62-65.

Laing DG, Panhuber H, Pittman EA, Willcox ME, Eagleson GK (1985) Prolonged exposure to an odor or deodorized air alters the site of mitral cells in the olfactory bulb. Brain Res 336:81-87.

Laissue PP, Reiter C, Hiesinger PR, Halter S, Fischbach KF, Stocker RF (1999) Three-dimensional reconstruction of the antennal lobe in Drosophila melanogaster. J Comp Neurol 405:543-552.

Manning A (1967) "Pre-imaginal" conditioning in Drosophila. Nature 216:338-340.

Martin KC, Kandel ER (1996) Cell adhesion molecules, CREB, and the formation of new synaptic connections. Neuron 17:567-570.

Masson C, Mustaparta H (1990) Chemical information processing in the olfactory system of insects. Physiol Rev 70:199-245.

McBride SM, Giuliani G, Choi C, Krause P, Correale D, Watson K, Baker G, Siwicki KK (1999) Mushroom body ablation impairs shortterm memory and long-term memory of courtship conditioning in Drosophila melanogaster. Neuron 24:967-977.

Milner B, Squire LR, Kandel ER (1998) Cognitive neuroscience and the study of memory. Neuron 20:445-468.

Moser MB (1999) Making more synapses: a way to store information? Cell Mol Life Sci 55:593-600.

Müller U (2000) Prolonged activation of cAMP-dependent protein kinase during conditioning induces long-term memory in honeybees. Neuron 27:159-168.

Nighorn A, Healy MJ, Davis RL (1991) The cyclic AMP phosphodiesterase encoded by the Drosophila dunce gene is concentrated in the mushroom body neuropil. Neuron 6:455-467.

Panhuber H (1986) The effect of long duration postnatal odour exposure on the development of the rat olfactory bulb. In: Ontogeny of olfaction (Breipohl W, ed), pp 127-137. Springer-Verlag.

Pinching AJ, Døving KB (1974) Selective degeneration in the rat olfactory bulb following exposure to different odors. Brain Res 82:195-204.

Pinto L, Stocker RF, Rodrigues V (1988) Anatomical and neurochemical classification of the antennal glomeruli in Drosophila melanogaster Meigen (Diptera: Drosophilidae). Int J Insect Morphol Embryol 17:335-344.

Rodrigues V (1988) Spatial coding of olfactory information in the antennal lobe of Drosophila melanogaster. Brain Res 453:299-307.

Rodrigues V, Buchner E (1984) [ $\left.{ }^{3} \mathrm{H}\right] 2$-Deoxyglucose mapping of odor- induced neuronal activity in the antennal lobes of Drosophila melanogaster. Brain Res 324:374-378.

Rose SP, Stewart MG (1999) Cellular correlates of stages of memory formation in the chick following passive avoidance training. Behav Brain Res 98:237-243.

Rubin BD, Katz LC (1999) Optical imaging of odorant representations in the mammalian olfactory bulb. Cell 96:725-736.

Sachse S, Rappert A, Galizia CG (1999) The spatial representation of chemical structures in the antennal lobe of honeybees: steps toward the olfactory code. Eur J Neurosci 11:3970-3982.

Sandoz JC, Laloi D, Odoux JF, Pham-Delegue MM (2000) Olfactory information transfer in the honeybee: compared efficiency of classical conditioning and early exposure. Anim Behav 59:1025-1034.

Schuster CM, Davis GW, Fetter RD, Goodman CS (1996) Genetic dissection of structural and functional plasticity. II. Fasciclin II controls presynaptic structural plasticity. Neuron 17:665-667.

Sigg D, Thompson CM, Mercer AR (1997) Activity-dependent changes to the brain and behavior of the honey bee, Apis mellifera (L.). J Neurosci 17:7148-7156.

Sterio DC (1984) The unbiased estimation of number and sizes of arbitrary particles using the disector. J Microsc 134:127-136.

Störtkuhl KF, Hovemann BT, Carlson JR (1999) Olfactory adaptation depends on the Trp $\mathrm{Ca}^{2+}$ channel in Drosophila. J Neurosci 19:4839-4846.

Strausfeld NJ (1999) A brain region in insects that supervises walking. Prog Brain Res 123:273-284.

Teillet MA, Watanabe Y, Jeffs P, Duprez D, Lapointe F, Le Douarin NM (1998) Sonic hedgehog is required for survival of both myogenic and chondrogenic somitic lineages. Development 125:2019-2030.

Tempel BL, Bonini N, Dawson DR, Quinn WG (1983) Reward learning in normal and mutant Drosophila. Proc Natl Acad Sci USA 80:1482-1486.

Todd JL, Baker TC (1999) Function of peripheral olfactory receptors. In: Insect olfaction (Hansson BS, ed), pp 67-96. Berlin: Springer-Verlag.

Tully T, Quinn W (1985) Classical conditioning and retention in normal and mutant Drosophila melanogaster. J Comp Physiol [A] 157:263-277.

West MJ (1999) Stereological methods for estimating the total number of neurons and synapses: issues of precision and bias. Trends Neurosci 22:51-61.

Winnington AP, Napper RM, Mercer AR (1996) Structural plasticity of identified glomeruli in the antennal lobes of the adult worker honey bee. J Comp Neurol 365:479-490.

Withers GS, Fahrbach SE, Robinson G (1993) Selective neuroanatomical plasticity and division of labor in the honeybee. Nature 364:238-240

Yuste R, Sur M (1999) Development and plasticity of the cerebral cortex: from molecules to maps. J Neurobiol 41:1-6.

Zhong Y, Wu C-F (1991) Altered synaptic plasticity in Drosophila memory mutant with altered cAMP cascade. Science 251:1562-1564.

Zhong Y, Wu C-F (1993) Differential modulation of potassium currents by cAMP and its long-term and short-term effects: dunce and rutabaga mutants of Drosophila. J Neurogenet 9:15-27.

Zhong Y, Budnik V, Wu C-F (1992) Synaptic plasticity in Drosophila memory and hyperexcitable mutants: role of cAMP cascade. J Neurosci 12:644-651. s 